\title{
La confrontation aux risques à proximité d'ouvrages industriels : le cas des pêcheurs à la ligne
}

\author{
Olivier Guillaume*
}

Sociologie, EDF Lab, Université de Versailles-Saint-Quentin-en-Yvelines, UMR PRINTEMPS, Guyancourt, France

Reçu le 25 juin 2017. Accepté le 24 septembre 2019

Nombre d'articles ont déjà été publiés dans NSS au sujet de la gestion des risques. Peu s'intéressent toutefois aux pratiques de loisirs de certains usagers qui se déroulent au sein de zones interdites et dangereuses - ici, en l'occurrence, une activité de pêche à la ligne aux abords d'un canal desservant une centrale hydroélectrique. La démarche compréhensive que déploie l'auteur lui permet d'appréhender l'expérimentation concrète des risques encourus par les pêcheurs, l'adaptation préventive de leurs comportements qui s'ensuit, ainsi que les ambiguïtés en matière de règles et d'interdits qui sont, à la fois, perçues et entretenues par les uns et les autres - individus, associations de pêcheurs, exploitant du canal, élus locaux - en vue d'établir des compromis acceptables dans des espaces confinés et discrets.

La Rédaction

Résumé - Attirés par de beaux salmonidés, la fraîcheur et la beauté des paysages, des pêcheurs à la ligne pratiquent leur loisir dans des zones de rivière ou de canaux classées dangereuses ou interdites car à proximité d'ouvrages industriels. Se confrontant aux risques des industries, sans les dénoncer voire en les minimisant, ils apprennent à en discerner les contours et développent des comportements de prévention qui peuvent les amener à transgresser des règles. Loin de se résumer à une seule explication individuelle, la confrontation aux risques et aux règles résulte de situations rendues ambiguës par des superpositions de lois ainsi que par le positionnement d'acteurs et d'institutions qui cherchent à préserver leurs activités et leurs relations avec les différents partenaires du territoire. Soumis aux limites des mesures classiques de réduction des risques, les différents acteurs du territoire tentent d'élaborer de nouvelles solutions en concertation.

Mots-clés : technologies / territoire / ressources naturelles / risques industriels / pêche à la ligne

Abstract - Confronting risk near industrial structures: the case of anglers. Attracted by the salmonids and the freshness and beauty of landscapes, anglers practice their hobby in river areas or channels classified as hazardous or prohibited due to their location near industrial facilities. Faced with industrial hazards which they neither negate nor minimize, they learn to discern limits and develop preventive behaviors that can lead them to transgress regulations. Confrontation with risks and rules, far from being merely an individual explanation, results from situations made ambiguous by overlapping laws and regulations and by the positioning of actors and institutions seeking to safeguard their activities and their relations with the various local stakeholders. Within the limitations imposed by conventional measures of risk reduction, the different local stakeholders seek to develop new solutions through negotiation.

Keywords: technologies / territories / natural resources / industrial risks / anglers

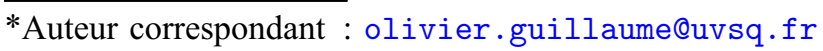


En 2009, sur les bords de la rivière Andrain ${ }^{1}$, deux pêcheurs situés dans une zone interdite de fréquentation par arrêtés préfectoraux sont surpris par une montée des eaux consécutive au lâcher ${ }^{2}$ d'une usine de production hydroélectrique. Ils sont secourus et hélitreuillés. Le premier vit depuis 30 ans dans la vallée, en connaît les risques et les arrêtés préfectoraux. Le second, également originaire de la région, s'est réfugié sur un îlot dans une zone autorisée puis a appelé les secours. Indépendamment de ces événements, des pêcheurs sont régulièrement aperçus dans la rivière au sein d'une zone interdite de fréquentation par des arrêtés préfectoraux. Et si la plupart évoquent leur méconnaissance des arrêtés et des risques, d'autres disent clairement les connaître et les braver. Dans une région du sud de la France, les berges d'un canal industriel privé dont l'eau alimente plusieurs industries sont fréquentées $\mathrm{du}$ grand public bien qu'interdites. Glissantes, elles pourraient provoquer la chute d'individus dans une eau froide et brassée où ils pourraient se noyer. Seuls les exploitants industriels du canal sont autorisés à s'y rendre. Pourtant, de nombreux pêcheurs s'installent en certains endroits des berges et banalisent les risques au travers de leur discours.

Ces exemples montrent que des riverains d'ouvrages industriels pratiquent parfois leur loisir au sein de zones classées dangereuses ou interdites. Ils se confrontent aux risques issus de ces industries, savent que ces risques peuvent leur porter atteinte mais minimisent la portée et l'incidence de leurs comportements. Plusieurs contributions en sciences sociales traitent des risques causés par les industries mais peu abordent ces cas rares. Celles axées sur la gestion interne des risques industriels mettent en lumière les causes organisationnelles et sociologiques ayant provoqué des accidents industriels (Perrow, 1984; Vaughan, 1999) ou les prévenant (La Porte, 1996). D'autres contributions retracent les controverses qui dénoncent l'implantation des ouvrages industriels (Lolive, 1997), les micromobilisations localisées (Chabbal, 2005) ou insistent sur les externalités de ces industries appréhendées comme des risques (Chateauraynaud et Torny, 1999; Borraz, 2008). Or ces travaux n'abordent ni la confrontation des individus aux risques, comprise ici comme la capacité d'y faire face et de s'y mesurer tout en s'y opposant, ni la minimisation de leurs comportements. Les théories de la perception permettent pour partie de comprendre la confrontation des individus aux risques. L'approche psychométrique montre ainsi qu'un risque sera considéré comme « familier » et minimisé ou, au contraire, amplifié

\footnotetext{
${ }^{1}$ Les noms propres sont fictifs pour rendre anonymes les lieux.

2 L'eau stockée dans un lac réservoir peut être acheminée jusqu'à une usine de production où elle est turbinée avant d'être relâchée dans une rivière en contrebas, engendrant la montée de ses eaux et de son courant.
}

et considéré comme un «danger», selon son occurrence, sa visibilité ou la connaissance de ses conséquences (Slovic, 1992). Mais cette approche exclut toutes situations concrètes et locales et ne montre pas la confrontation effective des individus aux risques.

Sortant du cadre industriel, d'autres travaux replacent les risques et leur gestion au sein de territoires. Les risques naturels majeurs peuvent être constitutifs de l'identité d'un territoire (Decrop, 2004), mais lorsqu'ils sont gérés par une poignée d'experts ne prenant pas en compte l'expertise locale des acteurs du territoire, ceuxci ne «croient» plus aux prévisions des experts et aux comportements rationnels qu'ils exigent d'eux (Decrop, 2004 ; Gentric et Langumier, 2009). Les controverses entre experts et acteurs sur les risques des territoires se résolvent grâce aux débats et aux négociations qui prennent en compte l'histoire sociale du territoire (Decrop, 2004) et les points de vue des parties prenantes (Thomi et Reynard, 2009). D'autres travaux éloignés des domaines industriels ou naturels expliquent les prises de risques volontaires des individus. Les adolescents testent ainsi leur nouveau corps, s'intègrent à de nouveaux groupes ou expriment le malaise d'une période transitoire par des prises de risques (Le Breton, 2002). Elles offrent aux adultes sportifs des sensations vertigineuses pour profiter «à fond» de leur jeunesse et de leur bonne santé (Soulé et Corneloup, 1998), se ressourcer en échappant à l'ennui (Le Breton, 2002) ou construire leur identité dans une société rationalisée et bureaucratisée (Lyng, 1990). Lors des activités sportives, les prises de risques et les transgressions des consignes permettent aux pratiquants d'acquérir de nouvelles connaissances ensuite recodées en consignes de sécurité (Raveneau, 2006) avant d'être diffusées au sein de la communauté de pratique (Boutroy, 2006).

\section{Problématique et méthode d'enquête}

Étrangères à un contexte industriel, les contributions citées montrent que les risques peuvent s'inscrire dans un territoire et son histoire et que les individus peuvent se confronter intentionnellement à eux. Mais qu'en est-il des risques industriels? Est-ce que les riverains de sites industriels peuvent se confronter délibérément aux risques générés par ces ouvrages? Et, si oui, comment expliquer ces comportements? Est-ce que le territoire, avec son histoire, ses acteurs, ses institutions, peut avoir une incidence sur la confrontation aux risques industriels?

Damien Femenias, Olivier Sirost et Barbara Evrard (2011) répondent pour partie à ces questions en retraçant les pratiques de loisirs nautiques dans la région de Rouen. Les auteurs montrent que plus les pratiquants sont en contact avec l'eau de la Seine, plus ils en perçoivent positivement la qualité, bien que l'estuaire du fleuve soit 
fortement exposé à la contamination chimique par les industries qui le bordent. Et plus un individu tombe à l'eau ou s'y baigne, plus il devient tolérant vis-à-vis de la qualité des eaux du fleuve, et plus il va accepter de se confronter aux risques sanitaires générés par les ouvrages industriels. Notre contribution poursuivra cette approche expliquant que la perception et la confrontation au risque industriel résultent d'un contact physique répété avec le milieu naturel. Mais elles résultent également d'acteurs institutionnels qui, sur un territoire, relaient ou non des messages, hiérarchisent les risques, en assurent ou non le contrôle.

Cette perspective collective et institutionnelle, complétant la compréhension de la confrontation aux risques, nous est apparue lors de recherches qualitatives menées en 2013 et 2014 pour le compte d'un exploitant industriel. Elles visaient en premier lieu à comprendre la perception et la confrontation des pêcheurs aux risques générés par des ouvrages industriels, le long de rivières et de canaux, puis en second lieu à proposer de nouvelles solutions de gestion des risques. Les études ont été consacrées aux pêcheurs à la mouche le long de rivières d'eaux vives, puis aux pêcheurs 《 au coup $»^{3}$ le long d'un canal.

Afin de comprendre les confrontations des pêcheurs à ces risques industriels, les recherches qualitatives compréhensives ont été privilégiées. Elles considèrent la réalité comme une construction humaine dont la signification résulte pour partie d'une interprétation du chercheur afin d'aller «au-delà » de ce que les données brutes disent a priori (Mukamurera et al., 2006; Blais et Martineau, 2006). Le monde social de la pêche à la ligne amateur nous étant inconnu au moment des recherches, une approche inductive «pure» a été privilégiée. La théorie et les cadres conceptuels ont émergé empiriquement des données recueillies grâce à une perspective ethnographique et interactionniste. Les données ont été analysées de manière thématique grâce à un processus de codification menant à une réduction des thèmes et à leur articulation (Blais et Martineau, 2006, p. 6-8) afin de proposer une théorisation ancrée (Glaser et Strauss, 2009).

L'étude qualitative présentée ci-dessous est axée sur les pratiques de pêche statique «au coup» le long d'un canal industriel. Elle est composée de vingt entretiens approfondis menés avec des représentants des fédérations départementales de pêches, des associations agréées de pêche et de protection des milieux aquatiques

\footnotetext{
3 La pêche dite "au coup» regroupe les techniques des «flotteurs», comme les bouchons, «au calé» et «au plombé». Statique et populaire, elle est souvent considérée comme plus simple que les pêches au lancer ou à la mouche (Roux, 2014, p. 359-360).
}

(AAPPMA) et des exploitants industriels locaux (managers, gestionnaires des risques, chargés de développement locaux). Des entretiens ont aussi été menés avec des pêcheurs rencontrés directement aux bords du canal ou grâce aux relations d'autres pêcheurs. Les entretiens ont été complétés par huit demi-journées d'observations directes de sorties de pêche, ponctuées de questions adressées aux pêcheurs au cours ou après les observations le long du canal, puis par l'analyse des documents internes aux clubs et de leurs sites internet. Des restitutions intermédiaires des analyses ont également été menées avec les protagonistes, permettant d'affiner les résultats.

Les parties suivantes décriront les activités de pêche à la ligne amateurs et celles des exploitants industriels le long d'un canal desservant des centrales de production hydroélectriques. Elles permettront de comprendre en partie pourquoi des riverains de sites industriels acceptent de se confronter aux risques, de pénétrer des zones interdites puis de minimiser leurs comportements. Leur confrontation aux risques résulte tout d'abord d'un apprentissage physique en situation. L'individu reconnaît alors les risques, les anticipe puis adopte des comportements qu'il estime de prévention, avant parfois de fréquenter des zones interdites. La confrontation aux risques et la transgression d'interdits sont ensuite accentuées par des situations juridiques qui apparaissent ambiguës, les actions des associations de pêche qui tentent d'accroître les zones de pêche et le nombre de leurs pratiquants, le comportement variable des forces de l'ordre et le positionnement des pouvoirs politiques locaux. Au final, les pêcheurs et leurs associations tentent de renégocier avec les exploitants industriels les règles qui encadrent les situations et les solutions pour gérer les risques.

\section{Pêcher dans un canal : une confrontation aux risques industriels}

Long de plusieurs dizaines de kilomètres, traversant des zones montagneuses et sauvages jusqu'à de grandes métropoles, le canal industriel de cette région du sud de la France a été créé plusieurs dizaines d'années auparavant en parallèle d'une rivière afin de contenir les eaux tempétueuses dont les brusques variations de niveau engendraient de terribles crues. Géré par une entreprise industrielle, le canal fournit dorénavant une ressource constante en eau pour l'irrigation de vergers et l'alimentation de plusieurs complexes industriels, notamment des centrales de production hydroélectriques. Le lit naturel de la rivière, parallèle au canal, est alimenté en eau par un débit dit «réservé ». Parce que le canal traversait des régions très peu peuplées lors de sa construction, quarante ans auparavant, ses concepteurs ne l'ont initialement pas doté de barrières tout son long, 
ne s'imaginant pas que la population locale se densifierait et que le canal serait investi.

Dans cette région rurale, les berges du canal sont dorénavant fréquentées par des arboriculteurs conventionnés avec l'entreprise exploitante et qui immergent leurs pompes dans l'eau pour irriguer leurs vergers. Elles le sont surtout par des joggeurs et, sur certaines portions, par des pêcheurs qui tentent d'attraper de beaux poissons, mais avec lesquels aucune convention n'existe. Or, cheminant le long des berges, ces pêcheurs pourraient tomber dans une eau froide, puis éprouver des difficultés à remonter en raison de bajoyers en béton longs et glissants. En quelques minutes, ils risqueraient de se retrouver en hypothermie ou d'être emportés par le fort courant avant d'entrer en collision avec les turbines ou les grilles des ouvrages industriels.

De nombreuses parades ont été mises en œuvre pour éviter des incidents faibles en nombre et en gravité. Il est ainsi formellement interdit aux personnes étrangères à l'entreprise gérant le canal de fréquenter les berges sans autorisation. La mesure est très régulièrement rappelée par des panneaux qui mentionnent la propriété privée. Des barrières et des rochers empêchent d'accéder en voiture sur les berges. Des panneaux précisent les risques de chute. Des filins agrémentés de bouées, dites «lignes de vie », fixées en amont et en aval des ponts permettent aux personnes qui seraient malencontreusement tombées à l'eau de s'en extraire grâce à des filins et des échelles spécifiques. Malgré ces mesures, on constate la présence de promeneurs et de pêcheurs sur les berges du canal, parfois à proximité des barrières et des panneaux.

Ce canal industriel aux berges en béton gris et sans ombre est fréquenté par les pêcheurs car il abrite de beaux salmonidés (truites, ombles, corégones) dont la présence résulte de l'interconnexion du canal avec des rivières et les lacs en amont ainsi que de lâchers artificiels d'alevins effectués par les sociétés de pêche ${ }^{4}$. Le volume conséquent d'eau froide courante et brassée ainsi que la présence en forte quantité de gammares ${ }^{5}$ permettent, en effet, une croissance rapide de belles truites pouvant atteindre $70 \mathrm{~cm}$ et $5 \mathrm{~kg}$, alors que la raréfaction de l'eau de la rivière détournée ne permet guère plus la croissance des salmonidés, au profit de poissons dits «blancs ${ }^{6}$, peu appréciés des pêcheurs de la région. Les routes longeant le canal rendent l'accès aisé à des berges stabilisées et débarrassées d'arbustes pour des pêcheurs âgés et locaux qui peuvent amener chaises, tables et glacières afin de se livrer à une pêche reposante, statique et d'attente.

\footnotetext{
${ }^{4}$ Les lâchers d'alevins directement dans le canal ont été réprouvés par les fédérations départementales de pêche voisines.

${ }^{5}$ De petites crevettes d'eau douce.

${ }^{6}$ Ablette, gardon, goujon, carpe, brème...
}

S'adaptant à leur environnement contraint par les aménagements industriels, les pêcheurs utilisent ainsi les affordances du canal, c'est-à-dire ses opportunités d'actions (Luyat et Regia-Corte, 2009), en étendant son usage à d'autres pratiques que celles initialement prévues (Akrich, 1998).

Enfin, il faut noter que, sur ce territoire rural, la pêche amateur «au coup» est un loisir ancestral pratiqué par des individus dorénavant plutôt âgés. Pouvant plus difficilement attraper de beaux salmonidés dans la rivière naturelle, en raison de la diminution de la ressource en eau et de berges plus accidentées et sauvages ${ }^{7}$, ils se rendent sur le canal parallèle.

\section{De la perception des risques à la transgression des règles}

Les pêcheurs fréquentent les berges qui leur sont interdites non pas en raison d'une méconnaissance des risques et des règles, mais parce qu'ils apprennent en situation à reconnaître certains risques et à développer des comportements qu'ils estiment de prévention.

\section{Expérience et perception du risque}

Sur le canal, la situation devient réellement dangereuse quand les pêcheurs tentent de récupérer, depuis les berges, de gros poissons. Dans ce cas, s'ils ne possèdent pas d'épuisettes suffisamment longues pour atteindre l'eau ou s'ils n'ont pas la force de remonter les poissons en tirant sur leur canne ou en moulinant le fil, les pêcheurs descendent le long des bajoyers afin d'aller chercher leurs prises. Ils risquent alors de tomber à l'eau, surtout s'ils sont âgés et fébriles. Les conséquences de l'accident sont d'autant plus graves que les pêcheurs âgés sont souvent seuls. Ils descendent d'autant plus le long des bajoyers que leur reconnaissance physique de la diversité des risques est limitée. Ne rentrant jamais dans l'eau, ils ne ressentent ni sa température basse ni la force du courant. Ils ne mentionnent alors comme risque que celui de tomber à l'eau sans insister sur la force du courant et de la température de l'eau qui sont les véritables risques naturels. «Les gens ne se mettent pas en danger. Ils ne se rendent pas compte que le courant est à un ou deux mètres ou qu'il y a du débit; ça ne les intéresse pas la technique. Le canal, c'est pourvu qu'il fasse soleil [...] pour les pêcheurs, c'est pas de danger. Ils se sentent à l'écart.» (membre d'une fédération de pêche).

\footnotetext{
La réduction du volume d'eau dans la rivière naturelle a élargi ses berges et a permis à une nature sauvage et touffue de se développer, limitant l'accès à l'eau.
} 
Par contre, observant les bajoyers et descendant parfois le long, les pêcheurs se confrontent au risque physique de glisser ${ }^{8}$. Ils adoptent alors des comportements qu'ils considèrent de prévention pour ce risque. Certains d'entre eux portent des crampons de glaciers ou des chaussures adaptées afin de ne pas glisser le long des bajoyers. D'autres se cramponnent à une corde attachée à leur voiture afin d'éviter de chuter lorsqu'ils descendent pour récupérer leurs prises. Des comportements qui révèlent leur mètis, cette intelligence pratique constituée de savoirs amateurs et utiles (Detienne et Vernant, 1986).

Au-delà d'une seule reconnaissance de signes tangibles, d'une expérience physique et concrète et de l'adoption de pratiques considérées de prévention, les confrontations des pêcheurs aux risques s'expliquent par l'ambiguïté des règles qui encadrent les situations de pêche et l'interrogation de leur légitimité.

\section{De la perception des risques à la perception des ambiguïtés}

La légalité de la pêche sur le canal est encadrée par plusieurs règles complémentaires mais qui peuvent apparaître contradictoires. Elles créent des situations ambiguës qui seront clarifiées par les pêcheurs grâce à leurs «compromis cognitifs» (Amalberti, 1996).

L'entreprise exploitant le canal mobilise la règle du droit de la propriété privée pour interdire de pénétrer sur les berges car le terrain lui est concédé par l'État pour ses activités de production. Cependant, en vertu des règles du droit de l'eau', l'entreprise ne peut pas interdire de pêcher dans les eaux du canal. En interconnexion avec la rivière, les eaux du canal sont considérées comme «libres». Elles relèvent alors du domaine public, concédant le droit de la pêche à l'État, et un acteur privé ne peut s'y opposer. La pêche dans le canal est donc théoriquement légale et peut s'effectuer, par exemple, depuis les ponts l'enjambant et non depuis les berges dont la gestion est concédée à l'entreprise. «Pour nous, dans les canaux, il y a des poissons qui peuvent rentrer. Ce sont donc des eaux libres. La réglementation de la pêche s'applique, donc le droit de pêche existe dans les canaux. » (fédération de pêche)

Par contre, le droit de la pêche oblige un pêcheur en eau douce et désirant pratiquer son loisir à adhérer à une association de pêche agréée, à acquitter sa cotisation auprès de celle-ci et à obtenir la permission de celui qui

\footnotetext{
${ }^{8}$ Certains admettent d'ailleurs être déjà tombés et avoir été secourus par un collègue.

${ }^{9}$ La loi n ${ }^{\circ} 64-1245$ du 16 décembre 1964 relative au régime et à la répartition des eaux et à la lutte contre la pollution est confirmée par la loi sur l'eau de 1992.
}

détient ou loue le droit de pêche. Propriétaire du droit de pêche en raison des eaux domaniales, l'État devrait alors louer son droit de pêche dans le canal aux AAPPMA ${ }^{10}$ locales au travers de baux de pêche. Mais l'État n'a jamais concédé et loué de baux de pêche sur les eaux domaniales du canal, notamment parce que les exploitants industriels ne le souhaitaient pas. Théoriquement légale sur le canal, la pêche n'y est techniquement pas autorisée, le canal étant dépourvu de baux de pêche dédiés.

L'autorisation de la pêche sur le canal est donc encadrée par trois sources de droit complémentaires, d'où une ambiguïté sur la légalité de la pêche en cet endroit. Pour Nicolas Lot (2008), l'ambiguïté renvoie à une situation où la concomitance et la rencontre de différentes sources de règles mobilisables par les acteurs rendent leur interprétation malaisée. Se matérialisant par une surabondance d'informations, l'ambiguïté provoque un état de confusion qui génère interrogation voire inquiétude, comme nous le précise un responsable d'association de pêche.

«-Responsable 1 : En plus, je vais vous dire, à l'époque, quand on avait les garde-pêches, ils s'étaient aperçus que quand on contrôlait un pêcheur, à la limite, il n'avait pas le permis, on ne pouvait pas poursuivre le gars en justice.

-Enquêteur: Pour quelle raison?

- Responsable 2: Parce qu'il n'y a pas de baux.

- Responsable 1: Il n'y a pas de baux ici. On n'a pas de baux.

-Enquêteur: Donc?

- Responsable 1: Donc, la justice disait: "Où vous l'avez attrapé ?". "On l'a attrapé sur le canal de l'entreprise en train de pêcher sans permis". "Vos baux ?". "On n'a pas de baux". On n'a pas de baux entre l'entreprise et nous pour nous autoriser la pêche ${ }^{11}$.

- Responsable 2: Ils n'ont jamais voulu signer.

-Enquêteur: On a le droit de pêcher, mais il n'y a pas de baux et on considère que c'est une propriété privée?

- Responsable 1: Oui, c'est tout ambigu! Oui, on est tout dans l'ambiguité. » (responsables association de pêche)

Les différentes sources de règles mobilisables obscurcissent la situation et conduisent les pêcheurs à tenir des positions hétérogènes ou changeantes. Pour un premier interviewé, la pêche et l'accès au canal sont autorisés à condition de ne pas y aller en voiture mais de

\footnotetext{
$\overline{{ }^{10} \text { Sur le domaine public }}$ l'État détient le droit de pêche et a la possibilité de le mettre en location. Il évalue un prix minimum et le droit de pêche est loué au plus offrant, souvent aux associations de pêche locales (AAPPMA).

${ }^{11}$ Les responsables de l'association de pêche rajoutent de la confusion en considérant que les baux de pêche doivent être concédés par l'entreprise exploitante. Les eaux appartenant à l'État, c'est à lui de concéder des baux de pêche.
} 
circuler à pied. Pour un second, la pêche sur le canal est rigoureusement interdite mais il avoue ne plus savoir quelle est la réalité après qu'on lui a soutenu le contraire. $\mathrm{Au}$ sein d'un groupe, certains expliquent qu'il est strictement interdit de pêcher sur le canal tandis que d'autres stipulent qu'il n'existe pas de limitations...

Afin de sortir de l'ambiguité d'une situation qui génère interrogations et doutes, les pêcheurs associent différents éléments afin de requalifier la situation pour ne plus considérer leur activité depuis les berges comme interdite, mais tolérée par l'entreprise exploitante. Ils procèdent pour cela en trois temps.

1) Ils soulignent tout d'abord la présence répétée et visible de pêcheurs sur le canal. «On m'a dit que c'était autorisé (de pêcher depuis les berges) mais j'ai vu que c'était privé. Mais comme j'ai vu que tout le monde pêche, je me suis dit que l'entreprise tolère.» (pêcheur $\mathrm{n}^{\circ} 1$, homme seul, 40 ans)

2) Ils mentionnent les rares contrôles des garde-pêches qui, lorsqu'ils interviennent, leur demandent leur permis sans réprimander leur présence sur les berges. "On ne sait pas trop, comme réglementation, c'est plutôt flou. Il y a très peu de gardes qui contrôlent le permis sur le canal.» (pêcheur $n^{\circ} 2$, homme seul 70 ans)

3) Ils insistent sur l'existence de lâchers de poissons dans le canal. «Du moment qu'ils lâchent le poisson, c'est autorisé» (pêcheur $\mathrm{n}^{\circ} 3$ ). «Je pense que c'est toléré.» (pêcheur $n^{\circ} 4$ )

En associant ces trois faits observés, ils concluent que leur activité depuis les berges est tolérée. Ils procèdent ainsi à un "compromis cognitif» (Amalberti, 1996). Quand un individu est confronté à des objectifs variés et peu clairs, à des informations conséquentes et parfois contradictoires et dont les ressources sont manquantes pour une compréhension optimale, il réduit la complexité de la situation en ne retenant que les informations essentielles à son action, en agrégeant celles cohérentes entre elles puis en supprimant les divergentes. La confrontation des pêcheurs aux risques et les accommodements par rapport aux règles s'expliquent enfin par le positionnement des associations de pêche, des forces de l'ordre et des pouvoirs publics qui, par leurs actions, entretiennent l'ambiguïté de la situation.

\section{L'entretien institutionnel des ambiguïtés}

Certains membres des associations locales de pêche ne découragent pas leurs adhérents de fréquenter les zones dangereuses et interdites car ils remettent en question la légitimité des règles définissant les situations de pêche sur le canal. Ils invoquent même un droit unilatéral d'y pêcher, sans restrictions, en compensation de la raréfaction de l'eau de la rivière qui, détournée en grande partie dans le canal, ne pourrait plus permettre la croissance des salmonidés.

« Il faut savoir que le fait d'avoir dérivé l'eau de la rivière et de l'avoir mise dans les canaux industriels, pour eux, ça les prive d'un potentiel de pêche sur le tronçon courtcircuité, et que quand finalement on vient leur dire: “Écoutez, il faut sortir du canal, parce que c'est dangereux, il y a du courant, l'eau est froide, les berges sont glissantes ", eux nous répondent que s'il n'y avait pas eu cet aménagement, si nous avions remis l'eau dans la rivière telle qu'elle était, c'est-à-dire sans débit réservé, avec un peu plus d'eau que le débit réservé, ils iraient pêcher en milieu naturel. » (exploitant industriel)

Cette compensation tacite et unilatérale conduit certains responsables d'associations de pêche à ne pas rappeler à leurs membres les interdits de fréquentation des berges et donc à ne pas dissuader leur présence.

«Nous, on ne leur dit pas que c'est interdit d'aller pêcher sur le canal. Mais on leur dit que c'est dangereux... mais ce n'est pas plus dangereux qu'ailleurs dans d'autres lieux. Personne dans l'association ne dit que c'est interdit. Par contre, sur nos dépliants, on ne fait pas de promotion des canaux. » (association de pêche)

Les «règles de contrôle» (Reynaud, 1989) qui définissent les situations de pêche et qui n'apparaissent pas toujours légitimes aux yeux des pêcheurs sont alors régulièrement transgressées. L'action sociale n'est en effet durable et légitime que si elle s'inscrit dans un cadre commun de règles constituées par échanges (Reynaud, 2003) et que ces règles construisent un sens à l'action sociale quand elles fournissent un but et une cohérence aux conduites de vie (Lallement, 2003, p. 232).

Les autres acteurs institutionnels de la région ne se mobilisent pas non plus considérablement pour faire respecter les interdits et mettre en avant ces situations risquées. Ainsi, les maires locaux, en raison de la diversité des risques à gérer sur leurs communes et avec des moyens constants, hiérarchisent implicitement les risques survenant selon leur gravité, leur occurrence et parfois leurs impacts auprès des électeurs. Et parmi les risques générés par l'eau, les crues et les inondations tangibles et aux potentiels de dégâts très marquants apparaissent politiquement et juridiquement comme beaucoup plus importantes que la présence de citoyens dans les zones interdites ou supposées dangereuses des berges du canal.

«Le maire est plus préoccupé par ça (les crues ${ }^{12}$ ) que par le canal. Surtout que le canal est en amont de sa commune, ça ne fait pas de vague, il n'en entend pas spécialement parler. Lui, il est plus intéressé par une bonne gestion des crues qui l'embêtent le moins possible que par ce canal. Mais ce n'est pas idiot de son point de vue. Si je priorise

\footnotetext{
12 Note du rédacteur.
} 
mes soucis, je regarde plus celui qui va concerner plus de monde, donc ces histoires de crues, et j'essaie de faire en sorte que ceux qui soi-disant peuvent faire quelque chose fassent quelque chose qui sauve de l'inondation mes administrés [...]» (exploitant industriel local)

Les exploitants industriels locaux, quant à eux, ne font pas non plus toujours appel aux forces de l'ordre quand ils constatent la présence de pêcheurs sur les berges du canal car ils n'ont pas reçu de consignes claires en ce sens et individuellement certains y rechignent.

«-[...] il n’y a pas de consigne lorsqu'on repère des pêcheurs sur le canal d'appeler la gendarmerie pour qu'ils viennent verbaliser?

- Non.

-Donc on ne le fait pas?

- Non.

- Du coup, il n'y a pas de verbalisation par la gendarmerie.

- Du coup, comme tu le dis, la gendarmerie ne va pas verbaliser spontanément. » (exploitant industriel local)

Un arrêté préfectoral qui interdirait tout loisir sur certaines portions du canal clarifierait la situation et donnerait davantage de poids aux forces de l'ordre pour intervenir. Mais les associations locales de pêche sont très vigilantes face à un tel projet et exprimeraient vivement leur mécontentement auprès des élus locaux. Revendiquant un nombre important d'adhérents qui sont autant d'électeurs, les associations locales de pêche sont des groupes structurés qui peuvent aisément se mobiliser et exercer des pressions sur les élus locaux, conduisant ces derniers à remonter les doléances des associations de pêche auprès du préfet afin d'éviter que de tels arrêtés ne soient pris.

«-Responsable 1: Mettons, en janvier-février, je vais faire mon assemblée générale, je leur dis: "Voilà, j'ai rencontré (X), ils nous ont annoncé que cette ouverture, on ne pouvait plus pêcher. On vous propose de manifester". Du bouche-à-oreille, on est quasiment sûr, entre les deux départements, de faire venir 500 pêcheurs.

-Enquêteur: Et ça, c'est une pression forte sur le système politique local?

-Responsable 1 : Je ne sais pas. Je pense que mon députémaire ici serait solidaire de nous. Mais purement électoral, non.

-Enquêteur: C'est un soutien politique important?

- Responsable 1: Oui.

- Responsable 2: Oui, ça fait 900 adhérents.

-Enquêteur: 900 sur une agglomération de?

-Responsable 1: De 7000. Nous, notre député-maire, il vient à toutes nos assemblées générales. On est une des plus grosses associations si on regarde en termes de licences. On est la plus grosse association de la ville [...] Je parle des associations sportives, culturelles, toutes les associations [...] Oui, on a du poids.» (responsables d'associations de pêche)

La confrontation des individus aux risques issus de l'industrie et aux règles de sécurité qui les encadrent ne s'explique donc pas par les seules expériences physiques des pratiquants au cours de leur activité de loisir. La compréhension de ce phénomène nécessite également de prendre en compte les intérêts et les stratégies d'action des associations qui organisent la communauté de pratique du loisir et celles des institutions locales qui supervisent le loisir sur le territoire par des normes et des contrôles. Dans le cas présent, la multiplicité des règles qui régissent la situation crée des ambiguiités qui ne sont pas totalement levées par les institutions. Elles disséminent indirectement des signes - comme l'absence de rétorsion par les gendarmes, le contrôle des seuls permis de pêche, les lâchers de poissons dans le canal par les sociétés de pêche...- lus et interprétés comme un affaiblissement des interdits et une légitimation de la tolérance de la pêche. Conscients d'être face à des situations peu satisfaisantes, exploitants industriels et associations de pêche tentent alors de négocier de nouvelles solutions pour entrouvrir les zones interdites.

\section{Vers la négociation de solutions coélaborées}

Sur le canal, associations de pêche et exploitants industriels tentent d'élaborer des zones de pêche sécurisées avec des barrières de protection. Cette solution s'est d'abord heurtée aux difficultés de déterminer les bons «porte-parole» des pêcheurs et de savoir les «intéresser» (Callon, 1986). Initialement désignés, les responsables départementaux des fédérations de pêche n'ont pas été en mesure de localiser les zones à sécuriser en raison de leur éloignement d'un canal qu'ils ne pratiquent pas et de leur souhait de ne pas imposer des emplacements aux présidents des associations de pêche locales. Ces derniers, en effet, sont leurs principaux soutiens et électeurs pour l'octroi des mandats départementaux. Désignés par la suite "porte-parole», les responsables des AAPPMA locales se sont emparés du projet car ils ont vu en ces zones une facilitation de la pratique de leur loisir, un accommodement pour leurs membres et une validation de la pêche sur le canal. En échange, ils se sont engagés à donner des consignes fermes pour que les pêcheurs ne sortent pas des zones mais refusent qu'elles s'accompagnent de l'interdiction de la pêche sur le reste du canal. 
L'évolution de la gestion des risques industriels résulte ici d'une consultation et d'une participation des utilisateurs et de leurs représentants afin de saisir leurs perceptions différenciées des risques et leurs pratiques multiples (Soulé et al., 2007) pour élaborer de nouvelles procédures (Kaufmann et al., 2004). La résolution du problème commun induit en effet une coopération et une convergence des intérêts afin de coélaborer des compromis acceptables issus «d'arènes du risque » (Renn, 1992).

Néanmoins, certains exploitants industriels locaux se sont interrogés sur l'efficacité de la solution proposée. Les zones de pêche sécurisées imposeraient en effet aux pêcheurs de se cantonner dans des environnements réduits alors que les habitués de ce loisir recherchent calme, sérénité et grands espaces. Et dans ces espaces réduits, les pêcheurs pourraient se gêner et tomber à l'eau, renvoyant ainsi la responsabilité de l'accident sur l'aménageur de l'espace de pêche qui serait l'exploitant industriel. Enfin, la création de ces aménagements pourrait être lue par les pêcheurs comme une normalisation de leurs pratiques, ce qui n'est pas l'avis de tous les exploitants, notamment ceux qui sont responsables de la sécurité de ces zones. N'ayant pas fait consensus parmi les industriels, la solution des zones sécurisées de pêche n'a finalement pas été adoptée, au profit d'une multiplication des «lignes de vie» en amont et en aval des ponts et des zones fréquentés sur le canal, permettant à des personnes malencontreusement tombées à l'eau de s'y accrocher et de remonter sur les digues grâce à une échelle.

\section{Conclusion}

Alors que les sciences sociales analysant les risques industriels insistent souvent sur les controverses et les mobilisations qui les dénoncent (Lolive, 1997; Chabbal, 2005), leurs externalités négatives (Borraz, 2008) ou les facteurs organisationnels et sociaux qui conduisent aux accidents (Perrow, 1984; Vaughan, 1999), notre contribution a voulu montrer que, dans certaines situations, des praticiens de loisirs se confrontent aux risques produits par les ouvrages industriels, qu'il s'agisse de risques sanitaires (Femenias et al., 2011) ou ici de sécurité. Leur confrontation aux risques, entendue comme une capacité à faire face aux risques tout en s'y mesurant, ne résulte pas d'une méconnaissance ou d'une perception déconnectée d'une situation concrète et locale (Slovic, 1992). Elle est en partie volontaire et résulte d'une expérimentation physique du risque, qui permet ensuite d'en percevoir les contours puis d'adopter des comportements estimés de prévention. Comme nous l'explique Gilles Raveneau (2006), les prises de risques et les transgressions des consignes - fréquenter les berges et descendre le long des bajoyers - permettent d'acquérir de nouvelles connaissances ensuite recodées, pour les pêcheurs et non les exploitants industriels, en consignes de sécurité (adopter des équipements et des chaussures particulières pour descendre le long des bajoyers).

La compréhension de la confrontation aux risques ne se réduit pas aux seules expériences individuelles et physiques des pratiquants de loisir. Elle nécessite de prendre en compte l'histoire du territoire (Decrop, 2004). Dans cette région rurale, la pêche des salmonidés est une activité ancienne et de sociabilité, qui, pour des pêcheurs plus âgés, peut aujourd'hui se révéler plus difficile à pratiquer dans la rivière naturelle. Enfin, la confrontation aux risques se comprend par la prise en compte des intérêts et des actions des associations et des institutions du territoire. Les situations juridiques ambiguës ne sont pas toujours clarifiées par certains représentants des associations de pêche qui veulent étendre la zone de pratique de leur loisir, et certains exploitants industriels locaux hésitent à faire appel aux forces de l'ordre quand ils constatent la présence de pêcheurs sur les berges du canal.

Cette situation insatisfaisante pousse les acteurs à négocier en tenant compte des points de vue des parties prenantes (Thomi et Reynard, 2009) afin d'élaborer de nouvelles solutions plus satisfaisantes. Ils tentent ainsi d'instituer des zones sécurisées de pêche afin d'assurer la coexistence des activités industrielles et de loisir en toute sécurité sur le canal. Mais la mise en œuvre de la solution n'est pas aisée. Elle pourrait être considérée comme un accord tacite de la pratique de la pêche sur le canal et rien ne garantit que tous les pêcheurs les respecteraient. Des solutions techniques plus anciennes, comme le déploiement de nouvelles «lignes de vie», sont alors privilégiées, auxquelles s'ajoute le rôle de vigilance et d'alertes des pêcheurs et de leurs associations en cas d'événements. Elles représentent des compromis acceptables de tous, produits dans des espaces «confinés et discrets » et non remis en question car la définition et la reconnaissance publiques des problèmes ne sont pas toujours aisées (Gilbert et Henry, 2012).

\section{Références}

Akrich M., 1998. Les utilisateurs, acteurs de l'innovation, Éducation permanente, 134, 1, 79-89, https://halshs. archives-ouvertes.fr/halshs-00082051.

Amalberti R., 1996. La conduite de systèmes à risques, Paris, Presses universitaires de France.

Blais M., Martineau S., 2006. L'analyse inductive générale: description d'une démarche visant à donner un sens à des données brutes, Recherches Qualitatives, 26, 2, 1-18, www. recherche-qualitative.qc.ca/documents/files/revue/edition_re guliere/numero $26 \% 282 \% 29 /$ blais et martineau final2.pdf.

Borraz O., 2008. Les politiques du risque, Paris, Presses de Science-po.

Boutroy E., 2006. Cultiver le danger dans l'alpinisme himalayen, Ethnologie française, 36, 4, 591-601, http:// dx.doi.org/10.3917/ethn.064.0591.

Callon M., 1986. Éléments pour une sociologie de la traduction. La domestication des coquilles Saint-Jacques 
et des marins-pêcheurs dans la baie de Saint-Brieuc, L'Année sociologique, 36, 169-208.

Chabbal J., 2005. Le risque invisible la non-émergence d'un problème public, Politix, 70, 2, 169-195, http://dx.doi.org/ 10.3917/pox.070.0169.

Chateauraynaud F., Torny D., 1999. Les sombres précurseurs : une sociologie pragmatique de l'alerte et du risque, Paris, EHESS.

Decrop G., 2004. La montagne, le hameau et le prophète de malheur. Histoire d'un risque moderne, Ethnologie française, 34, 1, 49-57, http://dx.doi.org/10.3917/ethn.041.0049.

Detienne M., Vernant J.-P., 1986 [ $1^{\text {re }}$ éd. 1974]. Les ruses de l'intelligence. La mètis des Grecs, Paris, Flammarion.

Femenias D., Sirost O., Evrard B., 2011. Les loisirs nautiques dans l'estuaire de la Seine: médiations territoriales, consciences du milieu, VertigO, Hors-série, 10, http://dx. doi.org/10.4000/vertigo.11576.

Gentric J., Langumier J., 2009. Inondations des villes, inondations des champs. Norme et territoire dans la prévention des inondations sur l'île de la Barthelasse (Avignon), Natures Sciences Sociétés, 17, 3, 257-265, http://dx.doi.org/10.1051/nss/2009040.

Gilbert C., Henry E., 2012. La définition des problèmes publics : ente publicité et discrétion, Revue française de sociologie, 53, 1, 35-59, http://dx.doi.org/10.3917/rfs.531.0035.

Glaser B.G., Strauss A.L., 2009. La découverte de la théorie ancrée. Stratégies pour la recherche qualitative, Paris, Armand Colin. Traduit de : Discovery of grounded theory. Strategies for qualitative research, Chicago, Aldine, 1967.

Kaufmann A., Perret H., Bordogna Petriccione B., Audétat M., Joseph C. 2004. De la gestion à la négociation des risques : apports des procédures d'évaluation des choix technologiques, Revue européenne des sciences sociales, XLII, 130, 109-120, http://dx.doi.org/10.4000/ress.419.

Lallement M., 2003. Régulation et rationalisation, in Terssac G. de (Ed.), La théorie de la régulation sociale de JeanDaniel Reynaud, Paris, La découverte, 231-249.

La Porte T.R., 1996. High reliability organizations: unlikely, demanding, and at risk, Journal of Contingencies and Crisis Management, 4, 2, 60-71, http://dx.doi.org/10.1111/ j.1468-5973.1996.tb00078.x.

Le Breton D., 2002. Conduites à risque: des jeux de mort au jeu de vivre, Paris, Presses universitaires de France.

Lolive J., 1997. La montée en généralité pour sortir du Nimby. La mobilisation associative contre le TGV Méditerranée, Politix, 39, 109-130, www.persee.fr/doc/polix_0295-2319_1997_ num_10_39_1687.

Lot N., 2008. Genèse, usages et maintien de l'ambiguïté dans l'organisation: l'exemple de la radioprotection. Thèse de doctorat de sociologie, Paris, Université Paris 9.
Luyat M., Regia-Corte T., 2009. Les affordances: de James Jerome Gibson aux formalisations récentes du concept, L'Année psychologique, 109, 2, 297-332, http://dx.doi.org/ 10.4074/S000350330900205X.

Lyng S., 1990. Edgework: a social psychological analysis of voluntary risk taking, The American Journal of Sociology, 95, 4, 851-886, http://dx.doi.org/10.1086/229379.

Mukamurera J., Lacourse F., Couturier Y., 2006. Des avancées en analyse qualitative: pour une transparence et une systématisation des pratiques, Recherches Qualitatives, 26, 1, 110-138, www.recherche-qualitative.qc.ca/documents/ files/revue/edition_reguliere/numero26\%281\%29/mukamu rera_al_ch.pdf.

Perrow C., 1984. Normal accidents: living with high-risk technologies, New York, Basic Books.

Raveneau G., 2006. La plongée sous-marine, entre neutralisation du risque et affirmation de la sécurité, Ethnologie française, 36, 4, 613-623, http://dx.doi.org/10.3917/ethn.064.0613.

Renn O., 1992. The social arena concept of risk debates, in Krimsky S., Golding D. (Eds), Social theories of risk, New York, Praeger, 179-196.

Reynaud J.-D., 1989. Les règles du jeu. L'action collective et la régulation sociale, Paris, Armand Colin.

Reynaud J.-D., 2003. Une théorie de la régulation pour quoi faire? in Terssac G. de (Ed.), La théorie de la régulation sociale de Jean-Daniel Reynaud, Paris, La Découverte, 399-446.

Roux F., 2014. Des pêcheurs distingués. L'espace des pratiques halieutiques, in Coulangeon P., Duval J. (Eds), Trente après La distinction de Pierre Bourdieu, Paris, La Découverte, 354-364.

Slovic P., 1992. Perception of risk: reflections on the psychometric paradigm, in Krimsky S., Goldind D. (Eds), Social theories of risk, New York, Praeger, 117-152.

Soulé B., Reynier V., Corneloup J., 2007. La communication préventive sur les risques. Le cas des stations de sports d'hiver en France, Communication, 26, 1, 79-107, http://dx. doi.org/10.4000/communication.754.

Soulé B., Corneloup J., 1998. Jeunes et prises de risque sportives. Vers une approche sociologique contextualisée, Corps \& culture, 3, http://dx.doi.org/10.4000/corpsetculture.471.

Thomi L., Reynard E., 2009. Gouvernance des risques hydrologiques dans un contexte urbain-le cas de la ville de Berne (Suisse), Espaces et sociétés, 139, 4, 123-140, http://dx.doi.org/10.3917/esp.139.0123.

Vaughan D., 1999. Technologies à hauts risques, organisations et cultures: le cas de challenger. Communication au colloque Risques collectifs et situations de crise, 11 octobre, Paris.

Citation de l'article : Guillaume $\mathrm{O}$. La confrontation aux risques à proximité d'ouvrages industriels : le cas des pêcheurs à la ligne. Nat. Sci. Soc. 28, 3-4, 239-247. 